\title{
Exposições ocupacionais por fluidos corpóreos entre trabalhadores da saúde e sua adesão à quimioprofilaxia*
}

\author{
OCCUPATIONAL EXPOSURE OF HEALTH CARE WORKERS TO ORGANIC \\ FLUIDS AND ADHESIONTO CHEMOPROPHYLAXIS
}

\section{EXPOSICIONES OCUPACIONALES POR FLUIDOS CORPORALES ENTRE TRABAJADORES DE LASALUD Y SU ADHESIÓN A LA QUIMIOPROFILAXIS}

\section{Clara Alice Franco de Almeida', Maria Cecília Cardoso Benatti ${ }^{2}$}

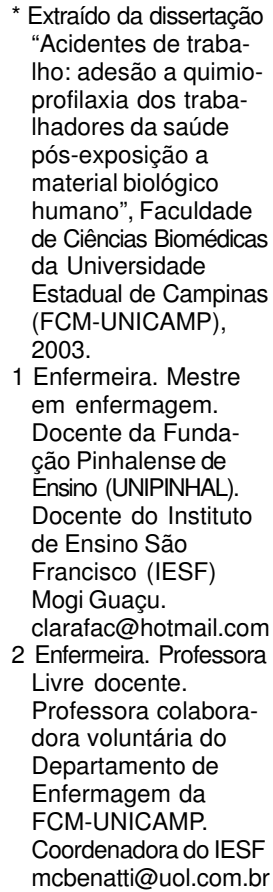

1 Enfermeira. Mestre em enfermagem. Docente da Fundação Pinhalense de Ensino (UNIPINHAL). Docente do Instituto de Ensino São Francisco (IESF) Mogi Guaçu.

clarafac@hotmail.com

2 Enfermeira. Professora

Livre docente.

Professora colabora-

dora voluntária do

Departamento de

Enfermagem da

FCM-UNICAMP

Coordenadora do IESF

mcbenatti@uol.com.br

\section{RESUMO}

Os objetivos do estudo foram investigar as características do acidente e do acidentado e avaliar a adesão à quimioprofilaxia e aos exames de controle e seguimento após o acidente ocupacional com risco de contaminação pelo vírus da imunodeficiência humana e vírus das hepatites B e C. Este é um estudo epidemiológico descritivo cujos dados foram coletados nas notificações realizadas em uma região administrativa da Secretaria de Estado da Saúde de São Paulo, Brasil, nos anos de 2000 e de 2001. Observou-se $7,3 \%$ de recusa à quimio-profilaxia contra o vírus da imunodeficiência humana por meio de anti-retroviral, e 40,6\% dos trabalhadores que a aceitaram não completaram o tratamento no tempo previsto de quatro semanas. Em razão desse diagnóstico, evidencia-se a necessidade de que as instituições envolvidas estabeleçam estratégias que possibilitem um aumento da adesão dos trabalhadores da saúde a esses cuidados.

\section{DESCRITORES}

Quimioprevenção.

Anti-retrovirais.

Acidentes de trabalho.

Pessoal da saúde.

Saúde ocupacional.

\section{ABSTRACT}

This study was aimed at investigating the characteristics of occupational accidents and of the workers that suffered them, and at evaluating the adhesion to chemoprophylaxis and to control and follow up tests after occupational accidents with risk of contamination by the human immunodeficiency virus and of the hepatitis B and C virus. This is a descriptive epidemiological study whose data was collected from the notifications of one of the administrative regions of the State of São Paulo's Secretary of Health between 2000 and 2001. It was observed $7.3 \%$ of refusals for chemoprophylaxis against human immunodeficiency virus by antiretrovirals, and that $40.6 \%$ of the care workers who accepted the chemoprophylaxis did not complete the treatment in the four weeks estimated for it. This diagnosis highlights the need for the institutions involved to establish strategies that make possible an increase in the adherence of health workers to these care procedures.

\section{KEY WORDS}

Chemoprevention.

Anti-retroviral agents.

Accidents, occupational.

Health personnel.

Occupational health.

\section{RESUMEN}

Los objetivos del estudio fueron investigar las características del accidente y del accidentado y evaluar la adhesión a la quimioprofilaxis y a los exámenes de control y seguimiento después del accidente ocupacional con riesgo de contaminación por el virus de la inmunodeficiencia humana y virus de las hepatitis B y C. Se trata de un estudio epidemiológico descriptivo cuyos datos fueron recolectados en las notificaciones realizadas en una región administrativa de la Secretaría de Estado de la Salud de Sao Paulo- Brasil, en los años 2000 y 2001 . Se observó un 7,3\% de rechazo a la quimioprofilaxis contra el virus de la inmunodeficiencia humana por medio de anti retroviral, y un $40,6 \%$ de los trabajadores que la aceptaron no completaron el tratamiento en el tiempo previsto de cuatro semanas. En razón de ese diagnóstico, se evidencia la necesidad de que las instituciones involucradas establezcan estrategias que posibiliten un aumento de la adhesión de los trabajadores de salud a esos cuidados.

\section{DESCRIPTORES}

Quimioprevención. Agentes antirretrovirales.

Accidentes de trabajo.

Personal de salud.

Salud laboral. 


\section{INTRODUÇÃO}

Os trabalhadores da área da saúde estão expostos aos mesmos riscos (químicos, físicos e ergonômicos) a que se sujeitam os demais trabalhadores brasileiros, acrescidos daqueles representados por agentes biológicos, uma vez que cotidianamente se expõem ao contato com sangue e outros fluidos orgânicos contaminados por uma variedade imensa de patógenos desencadeadores de doenças ocupacionais. Três delas são de grande relevância, principalmente após a década de 80 a AIDS, hepatite B e hepatite C. Geralmente tais doenças infecciosas são ocasionadas pela contaminação ocorrida por meio de acidentes com objetos perfurocortantes contendo fluidos corpóreos ou por respingos dos mesmos em mucosas e/ou pele lesada ${ }^{(1)}$.

Apesar do risco de adquirir infecções ao cuidar de pacientes contaminados por algum patógeno (vírus, bactéria ou outro microrganismo), por meio do contato com fluidos orgânicos ser bem conhecido e ter sempre existido, somente após a descoberta do vírus da imunodeficiência humana (HIV), como agente da Síndrome da Imunodeficiência Adquirida (AIDS) e a elucidação da sua forma de transmissão, as organizações estatais desencadearam esforços para diminuir as conseqüências dos acidentes com exposição ocupacional aos fluidos orgânicos potencialmente contaminados.

A transmissão da AIDS ocupacional tomou maior dimensão para os trabalhadores da saúde a partir do primeiro caso comprovado ocorrido com uma enfermeira em um hospital da Inglaterra, em $1984^{(2)}$.

A soroconversão dos trabalhadores da saúde por meio de exposição ocupacional aos vírus HIV, da hepatite B (HBV) e C $(\mathrm{HCV})$ tem sido reconhecida, comprovada e documentada ${ }^{(3)}$.

Muitos dos acidentes ocupacionais podem não resultar em infecção pelos vírus HIV, HBV e HCV mesmo que o pacientefonte esteja infectado. O risco é variável, devendo ser analisado todo o conjunto da situação. Existe ainda a possibilidade de diminuí-lo por meio da quimioprofilaxia (QP) para o HIV e HBV, não havendo essa possibilidade, por enquanto, para o HCV.

A QP para o HIV com os anti-retrovirais (ARV) surgiu em 1996 após estudos realizados pelos Centers for Disease Control (CDC) $)^{(4)}$ juntamente com estudos ${ }^{(5)}$ sobre a transmissão materno-infantil do HIV, que levaram em conta o efeito protetor da Zidovudina (AZT) nas gestantes, estendendo essa mesma possibilidade aos acidentes ocupacionais, na redução da viremia com o uso combinado de drogas anti$\mathrm{HIV}^{(6)}$. A partir desses estudos, os $\mathrm{CDC}^{(7)}$ elaboraram recomendação para que sejam administradas uma, duas ou mais drogas anti-retrovirais ao trabalhador por ocasião de acidente ocupacional.
Depois da introdução da terapia de anti-retrovirais, podese dizer que a AIDS é, nos dias de hoje, uma doença crônica controlável, pois os ARV atuam em diferentes pontos do ciclo do HIV, inibindo a multiplicação viral ${ }^{(8)}$.

Como as doenças crônicas demandam tratamento mais longo que as agudas, exigem também uma rotina, uma continuidade, uma regularidade no tratamento, de modo a serem controladas com eficácia. Por isso, alguns estudos têm utilizado as expressões adesão e aderência para designar essa regularidade do tratamento.

A adesão é a principal variável na qual os serviços de saúde podem intervir a fim de aumentar a eficácia da medicação e diminuir a possibilidade de resistência aos anti-retrovirais ${ }^{(8)}$.

Vários estudos ${ }^{(9-12)}$ têm sido desenvolvidos com o intuito de demonstrar a eficácia do tratamento prescrito a pacientes com diversos diagnósticos, tendo o olhar voltado para o comportamento do cliente, isto é, para o seguimento exato do tratamento proposto. No entanto, são poucas as publicações internacionais e nacionais sobre a adesão à QP em trabalhadores da saúde pós-exposição a material biológico.

Com esta investigação, as autoras esperam poder contribuir para a melhoria da saúde dos trabalhadores acidentados com exposição a fluidos corpóreos em seu posto ou local de trabalho.

Tiveram como objetivos investigar as características do acidente e das exposições ocupacionais dos trabalhadores da saúde acidentados a material biológico, com risco de contaminação (HIV, HBV e HCV), avaliar a adesão à quimioprofilaxia com anti-retrovirais (HIV) pós-exposição ocupacional a fluidos orgânicos humanos e comprovar a adesão dos trabalhadores acidentados aos exames protocolares de controle e/ou seguimento pós-exposição a material biológico.

\section{METODOLOGIA}

Trata-se de um estudo epidemiológico descritivo dos casos pós-acidente ocupacional com exposição a fluidos orgânicos que oferecem risco de contaminação por HIV, HBV e HCV. Inicialmente foi realizado um levantamento das fichas de notificação de acidentes biológicos com trabalhadores da saúde implantadas pela Secretaria de Estado da Saúde de São Paulo em 1999 ${ }^{(13)}$. Avaliou-se a adesão à conduta prescrita pelos serviços de saúde, no que diz respeito à quimioprofilaxia contra o HIV e aos exames de seguimento protocolares. Para a profilaxia contra o $\mathrm{HBV}$, não foi avaliada a adesão à vacina e/ou à imunoglobulina, pois o instrumento de notificação não contempla essa informação. No caso do HCV, ainda não existe recomendação de quimioprofilaxia. 
A aderência foi avaliada mediante dados contidos no instrumento de notificação e avaliação do acidente obtido através da informação direta e voluntária do acidentado ao serviço de saúde que o assistiu no momento do acidente e no seu seguimento, informando quantos dias o trabalhador utilizou os ARV contra o HIV e sobre a realização dos exames, contendo seus resultados.

Neste estudo adotou-se como definição de aderência receber $80 \%$ ou mais da medicação $(\mathrm{QP})$, o que equivale a 22 dias ou mais, independentemente do número de drogas prescritas, o que, por ser muito complexo, mereceria um estudo específico. As normas da Secretaria de Estado da Saúde em vigor indicam a QP por quatro semanas para todos os acidentados necessitados ${ }^{(9)}$.

Quanto à aderência aos exames de seguimento protocolares, avaliou-se a proporção dos acidentados que realizaram exames sorológicos no momento do acidente (momento zero), seis semanas, três meses e seis meses após o acidente independentemente do resultado sorológico do pacientefonte e da introdução da quimioprofilaxia ${ }^{(13)}$.

O estudo foi realizado no Departamento Regional (DIR) $\mathrm{XX}$, que representa uma das regionais de saúde do Estado de São Paulo, Brasil, junto ao Grupo de Vigilância Epidemiológica, com sede no município de São João da Boa Vista. A região é composta por vinte e um municípios e localiza-se na porção leste do Estado.

Foram utilizados documentos oficiais da vigilância epidemiológica da DIR XX encaminhados pelas vigilâncias epidemiológicas municipais, recebidos das instituições de assistência aos trabalhadores expostos a fluidos orgânicos humanos, em acidentes ocorridos nas instituições de saúde localizadas na área de abrangência da DIR XX de São João da Boa Vista.

A fonte de dados utilizada neste estudo foi a Ficha de Notificação de Acidentes Biológicos com Profissionais de Saúde padronizada pela Secretaria de Estado da Saúde de São Paulo.

A população do estudo é composta dos trabalhadores que sofreram acidentes nos quais houve exposição a fluidos orgânicos humanos, notificados no período de janeiro de 2000 a dezembro de 2001.

Foram considerados trabalhadores acidentados com exposição a fluidos orgânicos aqueles que tiveram sua notificação realizada pelo instrumento estadual oficial adotado, atendendo à recomendação vigente, que abrange todos os trabalhadores de instituições de saúde, incluindo os que recolhem lixo hospitalar e os que fazem a manutenção de equipamentos.

O estudo recebeu aprovação do Comitê de Ética em Pesquisa da Faculdade de Ciências Médicas da Universidade Estadual de Campinas, atendendo aos dispositivos das resoluções 196/96 e 251/97 do Conselho Nacional de Saúde (CNS).

\section{RESULTADOS E DISCUSSÃO}

Avaliou-se um total de 379 acidentes. Os dados dos trabalhadores da saúde acidentados e dos acidentes podem ser observados na Tabela 1.

Dos 379 acidentes estudados, a grande maioria $(78,9 \%)$ ocorreu com trabalhadores do sexo feminino. A explicação para os acidentes acontecerem mais entre as mulheres pode estar no fato da grande maioria dos trabalhadores da saúde serem mulheres ${ }^{(14)}$. A exposição percutânea foi o tipo de acidente mais notificado, representando $90 \%$ dos casos. O material orgânico de maior exposição foi o sangue, caso de 331 acidentados, o que equivale a $87,3 \%$ das ocorrências.

O principal agente causador dos acidentes foi a agulha com lúmen, representando $74,7 \%$ dos mesmos. O turno de maior ocorrência desses eventos foi o da manhã, das $7 \mathrm{~h}$ às $12 \mathrm{~h}(46,4 \%)$, seguido pelo da tarde, das $12 \mathrm{~h}$ às $17 \mathrm{~h} 59(34,6 \%)$, confirmando achados anteriores ${ }^{(15-16)}$ que demonstraram a maior ocorrência de acidentes nesse período do dia, no qual é administrado o maior número de medicações.

Nos resultados obtidos neste estudo, segundo as categorias ocupacionais dos acidentados, nota-se maior concentração entre os trabalhadores da equipe de enfermagem, responsáveis pela administração de medicamentos injetáveis, com $71,5 \%$ dos acidentes, seguidos pelos da limpeza, com $13,2 \%$ do total. Nesse último caso, os agentes causadores são agulhas e lâminas abandonadas no ambiente.

Em relação ao paciente-fonte observa-se que os acidentes aconteceram em situações em que as medidas preventivas nem sempre foram atendidas, tendo em vista que os pacientes-fonte são desconhecidos em 44,9\% dos acidentes. O maior problema parece estar relacionado ao descarte ou acondicionamento inadequado dos resíduos, pois os dados demonstram que a maioria $(79,4 \%)$ dos acidentes foi causada por agulhas (agulhas com lúmen e sem lúmen). Nesse caso, se o acidente tivesse ocorrido no momento do procedimento, o paciente-fonte não seria desconhecido.

Quanto à prescrição de quimioprofilaxia $(\mathrm{QP})$ com antiretroviral (ARV), os dados encontrados entre os 379 acidentados mostram que 165 casos $(43,5 \%)$ tiveram prescrição medicamentosa (QP) com ARV, distribuídos em 62 casos $(37,5 \%)$ com paciente-fonte conhecido e 103 casos $(62,5 \%)$ com paciente-fonte desconhecido. Entre os 165 acidentados com prescrição de QP, 12 recusaram a medicação, representando $7,3 \%$ de recusa.

Dos 379 acidentados, $165(43,5 \%)$ receberam indicação para QP com ARV, $53(32,1 \%)$ tiveram QP com vacina contra hepatite B e dois acidentados $(1,2 \%)$ receberam imunoglobulina humana contra a hepatite B. 
Tabela 1 - Caracterização dos trabalhadores da saúde acidentados com exposição a fluidos corpóreos da região de São João da Boa Vista, no período de 2000 a 2001

\begin{tabular}{|c|c|c|c|}
\hline Características & & $\mathbf{N}$ & $\%$ \\
\hline Acidentes notificados & & 379 & 100 \\
\hline \multirow[t]{2}{*}{ Sexo } & Feminino & 299 & 78,9 \\
\hline & Masculino & 80 & 21,1 \\
\hline \multirow[t]{6}{*}{ Tipo exposição } & Percutânea & 341 & 90,0 \\
\hline & Mucosa ocular & 14 & 3,7 \\
\hline & Pele íntegra & 11 & 2,9 \\
\hline & Mucosa oral & 9 & 2,4 \\
\hline & Pele não-íntegra & 3 & 0,8 \\
\hline & Ignorada & 1 & 0,2 \\
\hline \multirow[t]{3}{*}{ Material exposto } & Sangue & 331 & 87,3 \\
\hline & Outros fluidos & 20 & 5,3 \\
\hline & Não identificado & 28 & 7,4 \\
\hline \multirow[t]{6}{*}{ Agente causador } & Agulhas c/ lúmen & 283 & 74,7 \\
\hline & Outros instrumentos & 44 & 11,6 \\
\hline & Agulhas s/ lúmen & 18 & 4,7 \\
\hline & Lâminas & 15 & 4,0 \\
\hline & Vidros & 8 & 2,1 \\
\hline & Não identificado & 11 & 2,9 \\
\hline \multirow[t]{4}{*}{ Turno de ocorrência } & Manhã & 176 & 46,4 \\
\hline & Tarde & 131 & 34,6 \\
\hline & Noite & 47 & 12,4 \\
\hline & Não informado & 25 & 6,6 \\
\hline Categoria & Enfermagem & 271 & 71,5 \\
\hline \multirow[t]{7}{*}{ ocupacional } & Limpeza & 50 & 13,2 \\
\hline & Laboratorial & 16 & 4,2 \\
\hline & Odontológica & 12 & 3,2 \\
\hline & Administrativa & 11 & 2,9 \\
\hline & Médica & 8 & 2,1 \\
\hline & Manutenção & 4 & 1,1 \\
\hline & Outros setores & 7 & 1,8 \\
\hline \multirow[t]{2}{*}{ Paciente-fonte } & Conhecido & 209 & 55,1 \\
\hline & Desconhecido & 170 & 44,9 \\
\hline
\end{tabular}

Fonte: Vigilância Epidemiológica da DIR XX de São João da Boa Vista, 2003.

Os resultados sorológicos para o HIV são desconhecidos em $44,9 \%$ dos pacientes-fonte e $10,6 \%$ não haviam realizado o exame ou o resultado do exame era ignorado, o que perfaz um total de $55,5 \%$ dos pacientes-fonte sem conhecimento do status sorológico para o HIV (Tabela 2), aumentando o risco da infecção pelo HIV para os acidentados, pois nesta condição há maior necessidade de introdução da QP, desde que bem avaliado o acidente ocorrido.
Tabela 2 - Distribuição do resultado sorológico anti-HIV do paciente-fonte segundo a necessidade de introdução da QP com ARV aos trabalhadores acidentados da região de São João da Boa Vista, no período de 2000 a 2001

\begin{tabular}{lcccc}
\hline & Sem & Com & Total & \\
Resultado anti-HIV & QP & QP & N & \% \\
\hline Desconhecido & 67 & 103 & 170 & 44,9 \\
Negativo & 126 & 20 & 146 & 38,5 \\
Não Realizado/Ignorado & 19 & 21 & 40 & 10,6 \\
Positivo & 2 & 21 & 23 & 6,0 \\
\hline Total & 214 & 165 & 379 & $100 \%$ \\
\% & $56,5 \%$ & $43,5 \%$ & & \\
\hline
\end{tabular}

Fonte: Vigilância Epidemiológica da DIR XX de São João da Boa Vista, 2003. 
Os acidentes ocorridos com paciente-fonte conhecido totalizaram 209 casos. Em 165 deles, não foram realizados os exames relativos ao vírus da hepatite $\mathrm{B}$ e $\mathrm{C}$ no momento do acidente, representando um percentual de 78,9\% dos casos em que se desconhecia a condição do paciente-fonte em relação a esses vírus (Tabela 3 ).

Tabela 3 - Distribuição do resultado sorológico do paciente-fonte no momento do acidente, segundo o marcador sorológico correspondente, da região de São João da Boa Vista, no período de 2000 a 2001

\begin{tabular}{lcccccc}
\hline \multirow{2}{*}{ Resultado } & \multicolumn{2}{c}{ Anti-HIV } & \multicolumn{2}{c}{ HBsAg } & \multicolumn{2}{c}{ Anti-HCV } \\
& $\mathbf{N}$ & $\mathbf{0}$ & $\mathbf{N}$ & $\mathbf{\%}$ & $\mathbf{N}$ & $\mathbf{\%}$ \\
\hline Positivo & 23 & 11,0 & 1 & 0,5 & 6 & 2,9 \\
Negativo & 146 & 69,9 & 43 & 20,6 & 38 & 18,2 \\
Não realizado/ignorado & 40 & 19,1 & 165 & 78,9 & 165 & 78,9 \\
\hline Total & 209 & 100 & 209 & 100 & 209 & 100 \\
\hline
\end{tabular}

Fonte: Vigilância Epidemiológica da DIR XX de São João da Boa Vista, 2003.

Do total de acidentados, $276(72,8 \%)$ estavam com esquema vacinal completo no momento do acidente e 103 $(27,2 \%)$ com esquema incompleto ou ignorado para vacinação contra hepatite B (Tabela 4).

Tabela 4 - Distribuição dos acidentados segundo o esquema completo de vacinação contra hepatite B no momento do acidente, da região de São João da Boa Vista no período de 2000 a 2001

\begin{tabular}{lcc}
\hline Vacinação completa & $\mathbf{N}$ & $\mathbf{\%}$ \\
\hline Sim & 276 & 72,8 \\
Não & 90 & 23,7 \\
Ignorado & 13 & 3,5 \\
\hline Total & 379 & 100 \\
\hline
\end{tabular}

Fonte: Vigilância Epidemiológica da DIR XX de São João da Boa Vista, 2003.

Tabela 5 - Distribuição do tempo (em dias) da utilização dos ARV contra o HIV, da região de São João da Boa Vista no período de 2000 a 2001

\begin{tabular}{lcc}
\hline Dias & $\mathbf{N}^{\mathbf{0}}$ & \% \\
\hline$<22$ & 67 & 40,6 \\
$\geq 22$ & 98 & 59,4 \\
\hline Total & 165 & 100 \\
\hline
\end{tabular}

Fonte: Vigilância Epidemiológica da DIR XX de São João da Boa Vista, 2003.

Entre os 165 acidentados com prescrição à QP com ARV contra o risco de adquirir a infecção pelo HIV, 40,6\% dos acidentado não aderiram à $\mathrm{QP}$, pois o tratamento foi realizado por menos de 22 dias e $59,4 \%$ tiveram adesão ao tratamento, por utilizar a medicação por mais de 22 dias, sendo o ideal 28 dias. Em 94 casos, os acidentados receberam as drogas além do tempo necessário.
Tabela 6 - Distribuição do total de acidentados $(\mathrm{N}=379)$ segundo os períodos de realização dos xamessorológicos de seguimento protocolar, da região de São João da Boa Vista, no período de 2000 a 2001

\begin{tabular}{lccc}
\hline Período & Exames & $\mathbf{N}^{\mathbf{o}}$ casos & \% \\
\hline Momento zero & Anti-HIV & 346 & 91,3 \\
& HBsAg & 329 & 86,8 \\
& Anti-HBs & 233 & 61,5 \\
& Anti-HCV & 326 & 86,0 \\
$6^{\text {a }}$ semana & Anti-HIV & 234 & 61,7 \\
$3^{\text {o }}$ mês & Anti-HIV & 248 & 65,4 \\
$6^{\circ}$ mês & Anti-HIV & 294 & 77,6 \\
& Anti-HBV & 287 & 75,7 \\
& Anti-HCV & 288 & 76,0 \\
\hline
\end{tabular}

Fonte: Vigilância Epidemiológica da DIR XX de São João da Boa Vista, 2003.

Quanto aos exames protocolares de seguimento, entre o total de acidentados observa-se uma boa cobertura no momento do acidente, atingindo percentuais acima de $86 \%$ de execução do exame, com exceção do anti-HBs. No entanto, com o passar do tempo, os acidentados vão diminuindo a realização dos exames de seguimento, chegando ao final do cumprimento do protocolo com exames realizados em cerca de $76 \%$ dos casos (Tabela 6).

\section{CONCLUSÕES}

Neste estudo, apesar de não ter sido observada nenhuma soroconversão a qualquer dos patógenos estudados (HIV, HBV e HCV), observa-se que os acidentes aconteceram em situações em que as medidas preventivas nem sempre foram atendidas, tendo em vista que os pacientesfonte são desconhecidos em 44,9\% dos acidentes. O maior problema parece estar relacionado ao descarte ou acondicionamento inadequado dos resíduos, pois os dados demonstram que a maioria $(79,4 \%)$ dos acidentes foi causada por agulhas. Nesse caso, se o acidente tivesse ocorrido no momento do procedimento, o paciente-fonte não seria desconhecido.

$\begin{array}{rr}\text { Rev Esc Enferm USP } & \text { Exposições ocupacionais por fluidos corpóreos entre } \\ 2007 ; 41(1): 120-6 . & \text { trabalhadores da saúde e sua adesão à quimioprofilaxia } \\ \text { www.ee.usp.br/reeusp/ } & \text { Almeida CAF, Benatti MCC. }\end{array}$


Quanto à caracterização dos acidentes e das exposições ocupacionais dos trabalhadores da saúde, os dados demonstram que a grande maioria dos acidentes aconteceu com trabalhadores do sexo feminino $(78,9 \%)$. A categoria profissional mais acometida foi a equipe de enfermagem (71,5\%), tendo como principal turno de ocorrência dos acidentes o diurno. O tipo de exposição foi majoritariamente percutâneo, tendo como principal agente causador as agulhas com lúmen. O material orgânico da maioria das exposições foi o sangue $(87,3 \%)$.

Os dados demonstram que os trabalhadores de saúde acidentados estão com cobertura vacinal em torno de $72,8 \%$, lembrando que para ser considerado imunizado o indivíduo deve ter recebido o esquema completo (três doses) da vacina contra hepatite B.

Os resultados evidenciam o grande risco de adquirir a infecção pelo HBV a que estão expostos os acidentados, uma vez que $27,2 \%$ deles não tinham recebido o esquema completo de vacinação contra a hepatite B. Mesmo sendo os pacientes-fonte conhecidos, o teste para o vírus da hepatite $B$ não foi realizado ou não teve seu resultado conhecido em 78,9\% dos casos, aumentando assim a vulnerabilidade dos trabalhadores acidentados.

O risco de infecção através de um único ferimento com agulha para o vírus da hepatite $\mathrm{B}$ varia de acordo com a presença ou não do antígeno $e$ ( $\mathrm{HBe} A g)$ no sangue do infectante (paciente-fonte), sendo de $22 \%$ a $44 \%$ para aqueles com $\mathrm{HBeAg}$ sérico positivo e de $1 \%$ a $6 \%$ para os que apresentam HBeAg sérico negativo ${ }^{(17)}$.

$\mathrm{O}$ risco que os acidentes proporcionaram foram significativos tendo em vista a necessidade de introduzir a quimioprofilaxia (QP) para o HIV em 43,5\% dos acidentados.

A prescrição de QP para o HIV com anti-retrovirais aos acidentados foi maior ( 165 casos) do que a profilaxia contra o HBV (53 casos), apesar de o risco do HBV ser de $22 \%$ a 44\% ${ }^{(17)}$, ou seja, muito maior do que o risco da exposição ao HIV, que é de $0,3 \%$ para exposição percutânea ${ }^{(3)}$. No entanto, não pôde ser avaliada a conclusão da profilaxia para o $\mathrm{HBV}$, pois o instrumento oficial utilizado omitia essa informação.

\section{REFERÊNCIAS}

1. Gerberding JL. Management of occupational exposures to bloodborne viruses. N Engl J Med. 1995;332(7):444-51.

2. Needlestick transmission of HTLV-III from a patient infected in Africa. Lancet. 1984; 2(8416): 1376.

3. Cardo DM, Bell DM. Bloodborne pathogen transmission in health care workers. Risks and prevention strategies. Infect Dis Clin North Am. 1997;11(2):331-46.
Quanto à conclusão da prescrição da QP contra o HIV com ARV, observa-se que 98 acidentados $(59,4 \%)$ receberam as drogas prescritas em mais de vinte e dois dias, ou seja, tomaram pelo menos $80 \%$ da medicação prevista no protocolo (quatro semanas), sendo assim considerados aderentes.

Por ser a AIDS uma doença incurável, de grande impacto para a população mundial, cujos indicadores têm aumentado em toda as unidades de saúde, é imprescindível que os trabalhadores da rede estejam cada vez mais habilitados para atuarem preventivamente em seu cuidado ${ }^{(18)}$.

Nos exames protocolares, este estudo demonstra uma boa cobertura para o momento do acidente, atingindo percentuais acima de $86 \%$ de execução do exame. No entanto, a cobertura é baixa para os acidentados cujos pacientesfonte tiveram resultados conhecidos da sorologia para HIV $(80,9 \%), \operatorname{HBV}(21,1 \%)$ e HCV $(21,1 \%)$. Com o passar do tempo, por razão não contemplada neste estudo, os exames que deveriam ser realizados pelo acidentado vão deixando de ser feitos, atingindo no final do período de seguimento (sexto mês) taxas não superiores a 78\% para os três exames (anti-HIV, anti-HBV e anti-HCV).

Alguns estudos sobre intervenções a serem introduzidas no aperfeiçoamento da aderência de pacientes com doenças crônicas têm demonstrado que intervenções repetitivas e multifatoriais têm maior chance de aumentar a adesão ao tratamento $^{(19-20)}$.

Ao observar baixas taxas de adesão ao tratamento e aos exames de seguimento, podemos concluir a necessidade de todas as instituições envolvidas introduzirem novas estratégias a fim de aumentar a adesão à conduta prescrita aos trabalhadores de saúde acidentados com fluidos orgânicos.

Estudos anteriores demonstraram que as intervenções psicoeducacionais contínuas têm efeito mais duradouro, elevando a aderência ao tratamento ${ }^{(21)}$. Que esta condição seja uma sugestão aos gestores institucionais de saúde envolvidos em acidentes ocupacionais pós-exposição a material biológico.

4. Centers for Disease Control (CDC). Case control study of HIV serocon-version in health-care workers after percutaneous exposure to HIV-infected blood. France, United Kingdom, and United States, January 1988 - August 1994. MMWR Morb Mortal Wkly Rep. 1995;44(50):929-33.

5. Connor EM, Sperling RS, Gelber R, Kiselev P, Scott G, O‘Sullivan $\mathrm{MJ}$, et al. Reduction of maternal-infant transmission of human immunodeficiency virus type 1 with zidovudine treatment. $\mathrm{N}$ Engl J Med 1994;331(12):1173-80. 
6. New drugs for HIV infection. Med Lett Drugs Ther. 1996; 38 (972):35-7.

7. Centers for Disease Control and Prevention (CDC). Update: provisional Public Health Service recommendations for chemoprophylaxis after occupational exposure to HIV. MMWR Morb Mortal Wkly Rep. 1996;45(22):468-80.

8. Nemes MIB, Souza MFM, Kalichman AO, Grangeiro A, Souza RA, Lopes JF. Aderência ao tratamento por anti-retrovirais em serviços públicos de saúde no Estado de São Paulo [monografia na Internet]. Brasília: Ministério da Saúde; 2000 [citado 2003 fev. 1]. Disponível em http://www.aids.gov.br/final/ biblioteca/avalia1/home.htm

9. Alexandre NMC, Nordim M, Hiebert R, Campello M. Predictors of compliance with short-term treatment among patients with back pain. Rev Panam Salud Publica. 2002;12(2):86-94.

10. Cramer JA. Microelectronic systems for monitoring and enchancing patient compliance with medication regimens. Drugs. 1995;49(3):321-7.

11. Sollitto S, Mehlman M, Youngner S, Lederman MM. Should physicians withhold highly active antiretroviral therapies from HIV-AIDS patients who are thought to be poorly adherent to treatment? AIDS. 2001;15(2):153-9.

12. Chesney MA, Ickovics JR, Chambers DB, Gifford AL, Neidig J, Zwickl B, et al. Self-reported adherence to antiretroviral medications among participants in HIV clinical trial: the AACTG Adherence Instruments. AIDS Care. 2000;12(3):255-66.

13. São Paulo. Secretaria de Estado da Saúde. Recomendações e condutas após exposição ocupacional de profissionais de saúde. Bol Epidemiol Aids. 1999;17(1).
14. Machado AA, Costa JC, Gir E, Moriya TM, Figueiredo FC. Risco de infecção pelo vírus da imunodeficiência humana (HIV) em profissionais de saúde. Rev Saúde Pública. 1992; 26(1):54-6.

15. Silva VEF. Estudo sobre acidente de trabalho ocorrido em trabalhadores de enfermagem de um hospital de ensino [dissertação]. São Paulo: Escola de Enfermagem, Universidade de São Paulo; 1988.

16. Benatti MCC. Acidente do trabalho em um hospital universitário: um estudo sobre a ocorrência e os fatores de risco entre trabalhadores de enfermagem [tese]. São Paulo: Escola de Enfermagem, Universidade de São Paulo; 1997.

17. Stanford MP, Black TR, March LM, Holt DA, Campbell DH. Hepatitis B vaccination rates among staff at a District General Hospital. Med J Austral. 1995;162(20):304-6.

18. Thiengo MA, Oliveira DC, Rodrigues BMRD. Representações sociais do HIV/AIDS entre adolescentes: implicações para os cuidados de enfermagem. Rev Esc Enferm USP. 2005; 39(1):68-76.

19. Roter DL, Hall JA, Merisca R, Nordstrom B, Cretin D, Svarstad B. Effectiveness of interventions to improve patient compliance: a meta-analysis. Med Care. 1998;36(6):1138-61.

20. Haynes RB, Mckibbon KA, Kanani R. Systematic review of randomised trials of interventions to assist patients to follow prescriptions for medications. Lancet. 1996;348(9024):383-6.

21. Gifford AL, Bormann JE, Shively MJ. Effect of group HIV patient education on patient adherence to antiretrovirals: a randomised control trial [abstract 479]. In: Program and abstracts of the $8^{\text {th }}$ Conference on Retroviruses and Opportunistic Infections; 2001 Feb. 4-8; Chicago. Alexandria: Foundation for Retrovirology and Human Health; 2001. p. 186. 